\title{
Application of infrared thermography for validation of numerical analyses results of a finned cross-flow heat exchanger with non uniform flow of the agents
}

\author{
by T. Bury* and T. Kruczek*
}

*Institute of Thermal Technology, Silesian University of Technology, Gliwice, Poland

\begin{abstract}
The general aim of the work was determination of the influence of disturbances in velocity field of inlet air on the operation efficiency of heat exchanger under consideration. The inlet air velocity and temperature distributions, as well as the other characteristic parameters, were measured on a specially designed laboratory stand. The experimental results were then used as the input data in own computer code named HEWES. The outcomes of this calculations were compared with heat transfer estimated on the basis of measurements. An additional validation of the numerical results was performed by means of the infrared thermography measurements.
\end{abstract}

\section{Introduction}

The thermodynamic analysis of a ribbed cross-flow heat exchanger of the liquid-gas type for an example of a typical car radiator is the subject of the research. The heat transfer coefficients at the liquid and the gas side and the area of the heat transfer are the main parameters describing such a heat exchanger. Determination of the heat transfer coefficient at the liquid side does not make up a larger problem. The turbulent flow inside the pipes is a thoroughly examined case and the most of the existing empirical correlations give a comparable results [1]. The basic problem in computations of such a type of heat exchangers there is to calculate the heat transfer coefficient from the ribbed surfaces to a gas. Formulation of an universal correlation for the Nusselt number is not possible due to the diversity of constructions. The selection of the proper relationship for the specified geometry is also difficult, as well as the selection of the characteristic dimension. The diversity of the local values of the heat transfer coefficient resulting from the unequal flows of the agents through the exchanger additionally complicates the analysis [2]. The measurements were accomplished on the test station for research of the cross-flow heat exchangers at the Institute of Thermal Technology of the Silesian University of Technology to determine the form of the unequal air flux distribution at the inlet cross section of the considered radiator. Using the measurements results as the input data the heat transfer coefficient was numerically calculated. The final effect of these analyses is a computer code, called HEWES, for thermal analyses of the considered type of heat exchangers [3].

The initial arrangement of the test station has not allowed for validation of the code. The test station was modernized in the frameworks of the current research project and the validation procedure became possible. The total heat flux transported in the heat exchanger is the main comparing parameter, but the average heat transfer coefficient, air and water outlet temperature may be also compared. It was also assumed that the infrared thermography measurements of the outlet cross section temperature field may be useful for an additional validation of the HEWES code.

\section{Numerical model of the heat exchanger}

The mathematical model of the considered heat exchanger was worked out taking into account the following simplifying assumptions:

- steady state conditions,

- one-dimensional agents flow,

- no internal heat sources,

- radiation is neglected,

- heat losses are neglected,

- heat flow is normal to a boundary,

- real rib is replaced with a round or a plate-elliptic rib of the same surface.

It was also assumed that the air inflow is non-uniform and the water inflow may be non-uniform. An influence of temperature on thermal properties of the agents was taken into account too.

The analysed real cross-flow heat exchanger was replaced with a model rectangular heat exchanger. The model was then divided onto elementary fragments (see figure 1). Each fragment represents a recurrent element of the real heat exchanger - a single tube with the rib [3]. 


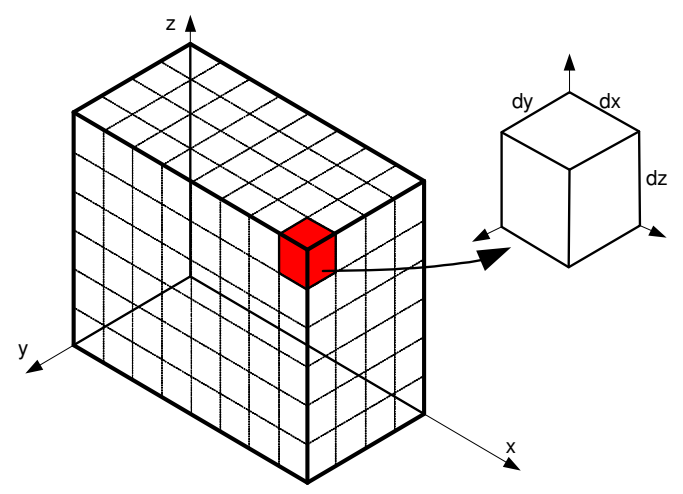

Fig. 1. Model heat exchanger and the recurrent fragment

The energy balance equations for each fragment constitute the mathematical basis of the model. Assuming that the water flows along the $X$ axis and the air flows along the $Y$ axis the energy balance for a recurrent fragment may be written as follow:

$$
d \dot{Q}=-\dot{m}_{w} c_{p w} \frac{\partial T_{w}}{\partial x} d x=\dot{m}_{a} c_{p a} \frac{\partial T_{a}}{\partial y} d y=\alpha_{a}\left(T_{m}-T_{a}\right) d A
$$

where $\alpha_{a}$ is an average heat transfer coefficient at the gas side for all the ribbed surface and $T_{m}$ is the average temperature of rib and pipe surface.

The control volume method based model of heat transfer for the recurrent fragment of the heat exchanger was worked out to calculate the average temperature of the ribs and tube outer surface. The detailed description of the model and equations can be found in [3].

The parameters calculated with the model of the recurrent fragment are: outlet and average temperature of the water flowing in the pipe, average temperature of the air, average temperature of the rib and the pipe surface, average values of the heat transfer coefficients at the gas side and the heat flux transported in the recurrent fragment. The heat transfer coefficient from the hot water to the pipe is computed from Colburn's formula [1].

The heat transfer coefficient at the gas side may be determined on the way of the numerical simulations for a numerical model of the recurrent fragment of the considered heat exchanger [4] or may be computed from one of available Nusselt number correlations.

The calculation procedure for the whole exchanger model is iterative and it is repeated for all the recurrent fragments of the considered heat exchanger. First, the air temperature increase in the analysed fragment is assumed. Next, the heat transfer coefficients for the water and the gas sides are calculated as well as the rib and pipe surface average temperature. The heat flux transported in the recurrent fragment is then computed and the accuracy criterion is checked. If the criterion is satisfied the procedure is realized for the next fragment. If the criterion is not fulfilled the described procedure is then repeated for the given recurrent fragment till the demand accuracy is achieved.

\section{Analyses of experiments and numerical simulations}

\subsection{The laboratory stand}

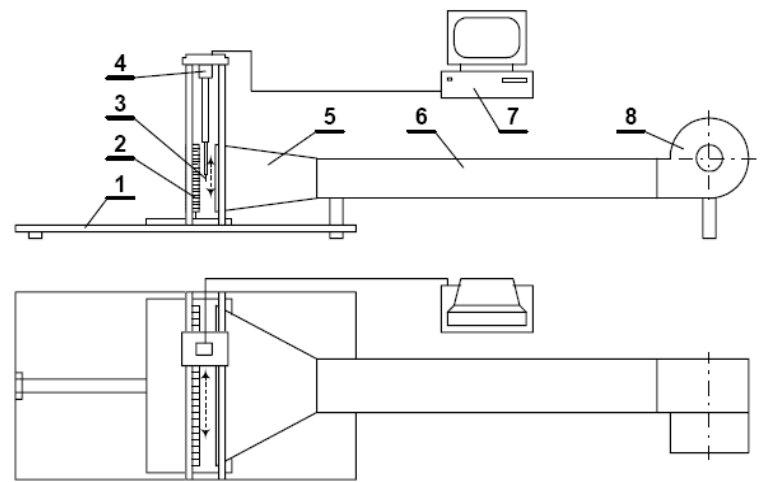

Fig. 2. The diagram of the laboratory stand - air supply module - (1 - support plate, 2 - considered heat exchanger, 3 - thermo-anemometric sensor, 4 - measuring probe, 5 - diffuser, 6 - air channel, 7 - control computer, 8 - fan) 
The simplified scheme of the laboratory experimental stand is shown in figures 2 and 3 . The temperature and velocity of cooling air at selected nodes of measuring net were continuously measured during the experiments. These parameters were measured by means of a thermo-anemometric sensor. The shifting of the sensor was controlled by a computer and the measuring results were recorded by computer as well. Additionally other parameters were measured as inlet and outlet water temperature, flow rate of water and total flow rate of air.

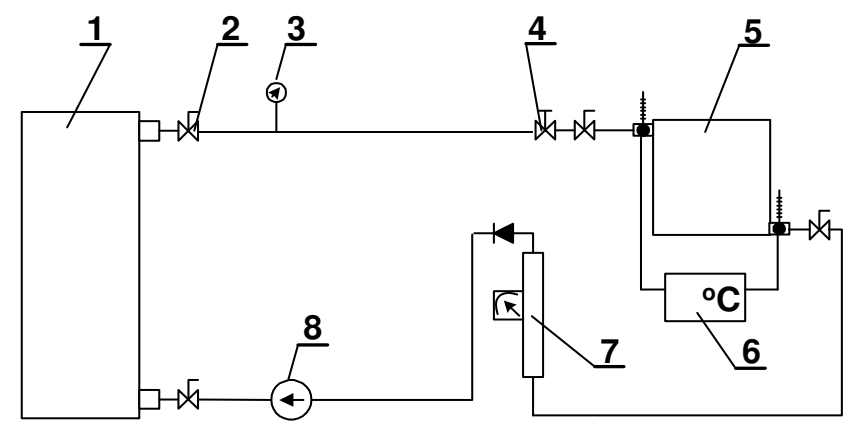

Fig. 3. The diagram of the laboratory stand - water supply module (1 - electric heater, 2 - cut-out valve, 3 manometer, 4 - control valve, 5 - heat exchanger, 6 - water temperature measuring system, 7 - flow meter, 8 pump)

Three parameters are assumed as independent and may be set by a researcher: the air and water streams and the inlet water temperature.

\subsection{Measurement procedure and results}

The measuring task must be defined before an experiment is started. The task information contains a thermo-anemometric sensor characteristic and the measuring net definition (coordinates of the measuring knots). The measurements were started after the steady state condition was achieved.

The air velocity and its temperature distribution at the inlet to the exchanger are ones of the most important experimental results. These information are used for computations of the heat transfer coefficient and for determination of the inequality factors. The distribution of the air velocity at the inlet cross-section of the cooler for one of the measuring series is presented in figure 4. An exemplary temperature distribution at the exchanger outlet is shown in figure 5. The temperature distribution information is used for calculations of the average inlet and outlet temperatures.

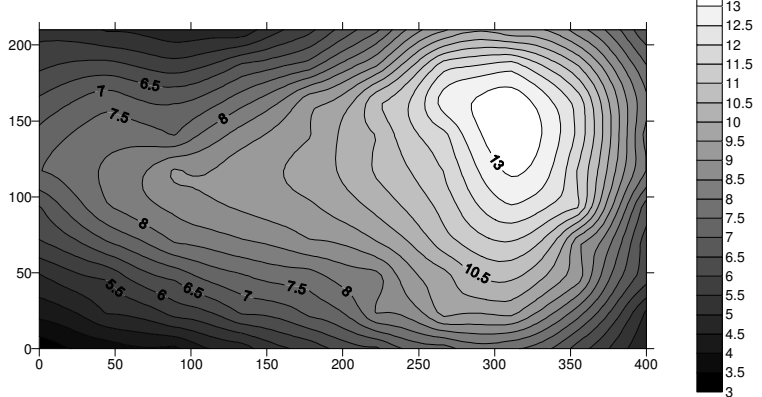

Fig. 4. Distribution of the velocity of the air at the inlet cross section of the car cooler for experiment $\mathrm{T} 2, \mathrm{~m} / \mathrm{s}$

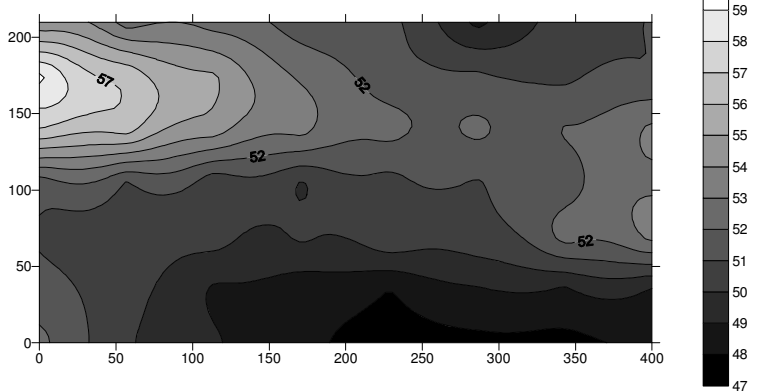

Fig. 5. Distribution of the temperature of the air at the outlet cross section of the car cooler for experiment $\mathrm{T} 4, \stackrel{\circ}{\mathrm{C}}$

The selected experimental data from these measurements are gathered in table I.

Table 1. Selected results of measurements

\begin{tabular}{|c|c|c|c|c|}
\hline \multirow{2}{*}{ Parameter } & \multicolumn{4}{|c|}{ Experiment } \\
\cline { 2 - 5 } & $\mathrm{T} 1$ & $\mathrm{~T} 2$ & $\mathrm{~T} 3$ & $\mathrm{~T} 4$ \\
\hline Air volumetric flow, $\mathrm{m}^{3} / \mathrm{s}$ & 0,78 & 1,25 & 0,78 & 1,25 \\
\hline Water volumetric flow, $\mathrm{m}^{3} / \mathrm{s}$ & $4,5 \cdot 10^{-4}$ & $4,5 \cdot 10^{-4}$ & $4,5 \cdot 10^{-4}$ & $4,5 \cdot 10^{-4}$ \\
\hline Air inlet temperature, ${ }^{\circ} \mathrm{C}$ & 33,01 & 34,12 & 30,65 & 34,51 \\
\hline Air outlet temperature, ${ }^{\circ} \mathrm{C}$ & 47,04 & 43,60 & 57,04 & 51,34 \\
\hline
\end{tabular}




\begin{tabular}{|c|c|c|c|c|}
\hline Water inlet temperature, ${ }^{\circ} \mathrm{C}$ & 58,4 & 59,1 & 78,7 & 78,6 \\
\hline Water outlet temperature, ${ }^{\circ} \mathrm{C}$ & 50,9 & 50,3 & 65,1 & 63,2 \\
\hline
\end{tabular}

\subsection{Code validation}

The validation of the model has been performed by means of the comparison of the experimental results with outcomes of the code. Four parameters have been compared:

- total heat flux transported in the exchanger,

- average heat transfer coefficient,

- average water temperature,

- $\quad$ average air temperature.

Table 2. Selected results of comparative analysis

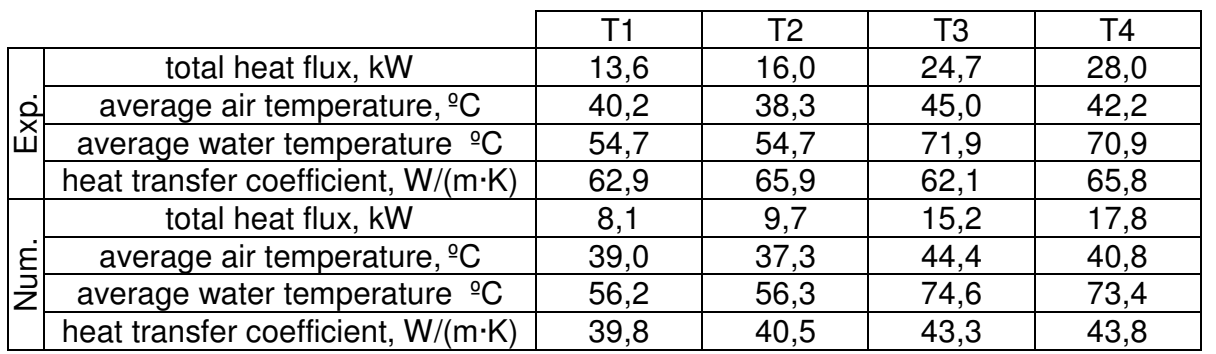

Selected results of the comparative analysis are shown in table 2. It can be observed that numerical values of the total heat flux are much smaller than the experimental ones. The way of the heat transfer coefficient determination has been indicated as the reason of these discrepancies. The results of performed initial sensitivity analysis show that the heat transfer coefficient may be not only one reason of the differences [5]. Relatively large change of the heat transfer coefficient values caused visible but not significant change of the total heat flux. A further works in this field are in progress at the moment.

The infrared thermography was decided to be used for extending of the range of the validation procedure. The aim of these measurements was to get data for comparison of numerically determined distribution of temperature at the outlet of the exchanger.

\section{Infrared thermography measurements}

The thermographic measurements have been done using ThermaCAM SC2000 camera. First measurements have been done with perpendicular arrangement of the camera in respect to the investigated surface, and they shown almost even distribution field of the temperature (see figure 6).

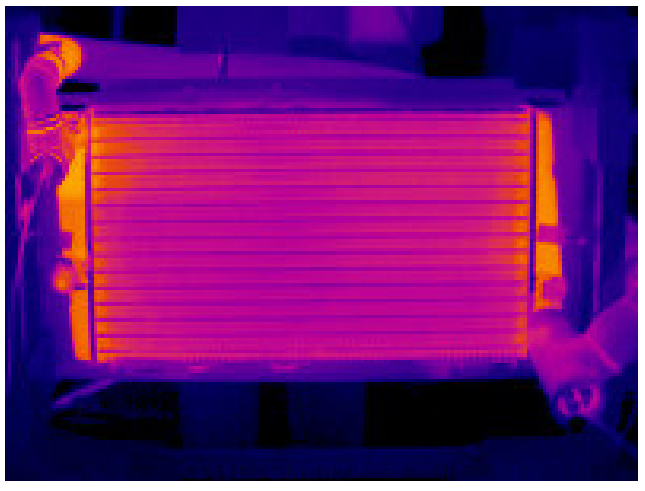

Fig. 6. Distribution of temperature at the outlet of the heat exchanger - exemplary results with the perpendicular arrangement of the camera in respect to the investigated surface
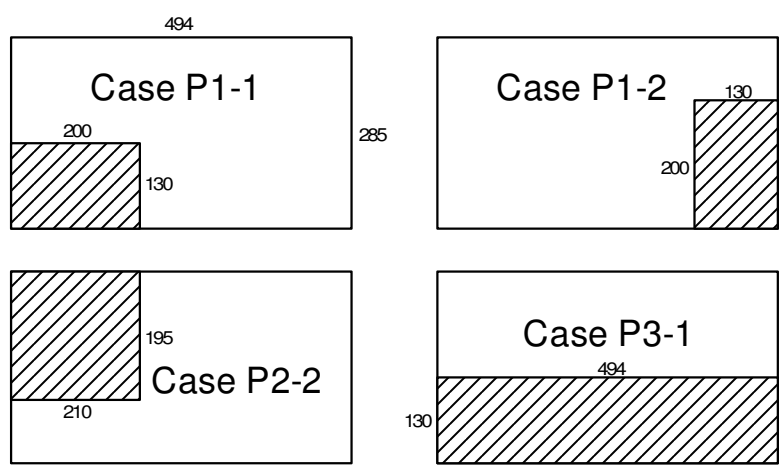

Fig. 7. The diaphragms localization and sizes for a selected cases

Because the results shown above can not be interpreted unambiguously they were not used for validation. The further infrared thermography measurements have been done with using different arrangements of the camera. The best results were obtained using sharp angle arrangements. The most measurements have been also realized with additional disturbance inserted in front of the exchanger in form of a diaphragm. The basic aim 
of this measurements was determination of the influence of disturbances in velocity field of inlet air on the operation efficiency of heat exchanger under consideration.

A cardboard diaphragms were used to block the air inflow to the heat exchanger in a part of the inlet crosssection. Diagrams showing a selected diaphragms localization and sizes are shown in figure 7. The diaphragms used during measurements allowed to cover from 18,5 up to $46 \%$ of the total inlet cross-section area.

A reference cases were investigated before the air inflow was choked. These measurements were the basis for evaluation of the influence of the air flow disturbances on the heat exchanger operation efficiency. The results of analysis are gathered in table 3. The total heat flux was calculated based on the water enthalpy drop. The same cases were also simulated by the HEWES code. The authors, remembering the significant differences described earlier, wanted to check if the code is able to predict the heat exchanger efficiency drop.

Table 3. Selected results of comparative analysis

\begin{tabular}{|c|c|c|c|c|c|}
\hline \multirow{2}{*}{ Case } & \multirow{2}{*}{\begin{tabular}{c} 
Reference \\
value of the \\
\cline { 3 - 6 }
\end{tabular}} & \multicolumn{2}{|c|}{ Experiment } & \multicolumn{2}{c|}{ HEWES } \\
\cline { 3 - 6 } & Heat flux, W & $\begin{array}{c}\text { Relative } \\
\text { heat flux } \\
\text { drop, \% }\end{array}$ & Heat flux, W & $\begin{array}{c}\text { Relative } \\
\text { heat flux } \\
\text { drop, \% }\end{array}$ \\
\hline P1-1 & 12079,2 & 10871,3 & 10,0 & 8377,4 & 13,5 \\
\hline P1-2 & 12079,2 & 10146,5 & 16,0 & 7651,1 & 21,0 \\
\hline P2-2 & 12079,2 & 9904,9 & 18,0 & 7215,3 & 25,5 \\
\hline P3-1 & 37580,2 & 28568,6 & 24,0 & 20146,6 & 32,3 \\
\hline
\end{tabular}

It may be noticed that the differences between the total heat flux obtained from experiments and computations are still significant. The HEWES code also overestimated the relative heat flux drop for the considered cases.

The infrared thermography measurements were realized during investigations of the considered cases. Results of these measurements were then compared with numerically determined temperature field at the heat exchanger outlet cross-section. First the reference cases were analysed. The exemplary results for such case are presented in figures 8 and 9. To make the infrared camera images more useful for comparison the original temperature scale was changed. One may observe in fig. 8 that there are at least two pipes where the water flow is very small. This effect is invisible in the numerical results of course, because a uniform water flow through all the pipes was assumed - see figure 9. Analysing temperature fields shown in figures 8 and 9 it can be observed than the numerically predicted temperature values are a little bit lower than the experimental ones. The differences reach up to $5 \mathrm{~K}$ and are a relatively large. It is very probable that the method of determination of the pipes wall and rib surface average temperature may be another reason for the differences revealed during validation. Moreover, the qualitative discrepancies are also visible - the isotherms shape is quite different.

The selected results for the cases with the diaphragms are shown in figures 10 to 12 . The diaphragms localizations are clearly visible on thermographic pictures. It may be noted that in the area covered by these diaphragms there are almost no water temperature drop considering the experimental results.

Only one numerically obtained temperature field is presented here due to limited space. Analysing results shown in figure 11 one may note that the border of the area covered by the diaphragm is not as sharp as in the thermographic picture (see figure 10). Despite of this the conformity of the experimental and numerical results for this case is even better than for the reference case presented earlier.

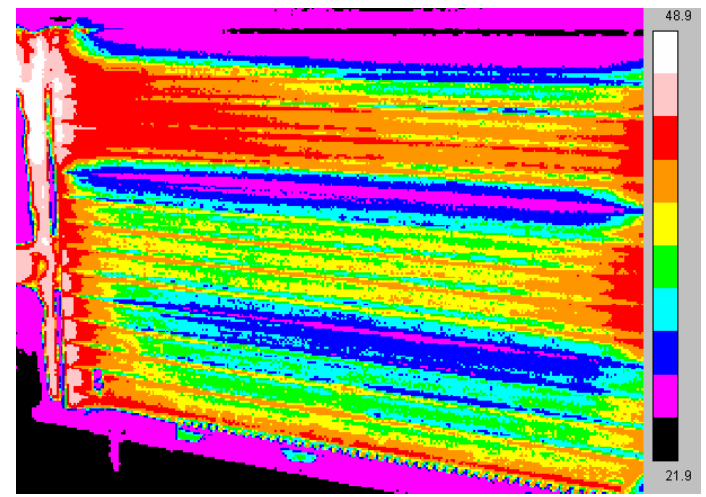

Fig. 8. The temperature field at the heat exchanger outlet cross-section - reference case, infrared measurement

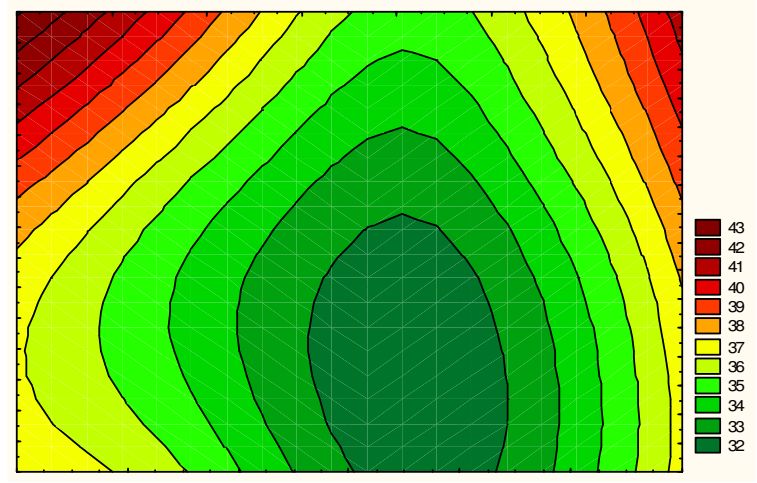

Fig. 9. The temperature field at the heat exchanger outlet cross-section - reference case, HEWES 


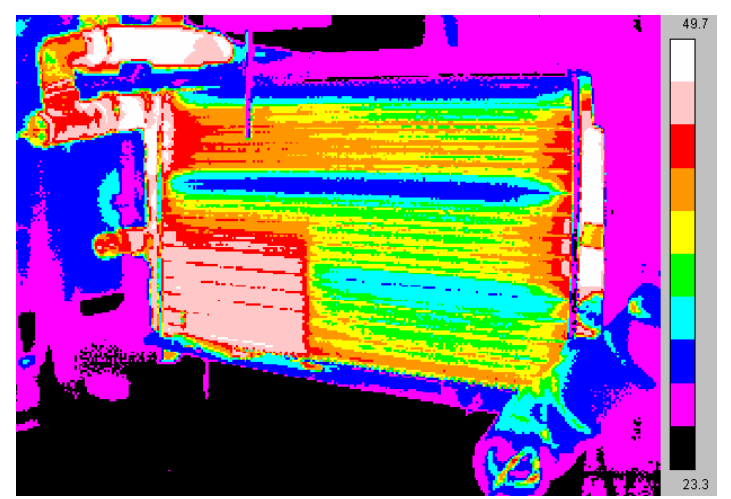

Fig. 10. The temperature field at the heat exchanger outlet cross-section - case P1-1, infrared measurement

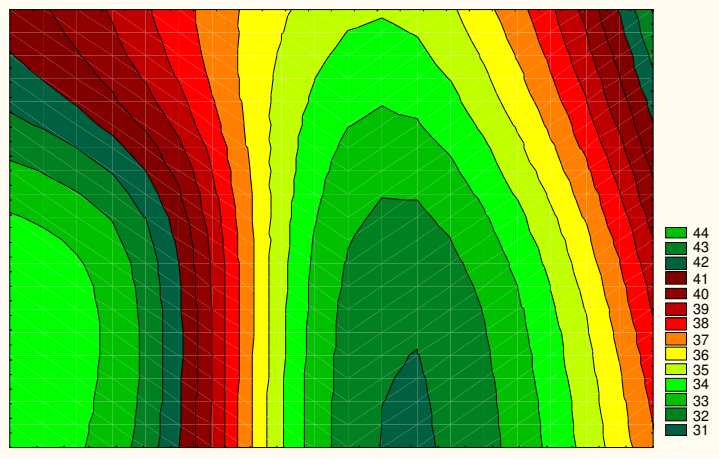

Fig. 11. The temperature field at the heat exchanger outlet cross-section - case P1-1, HEWES

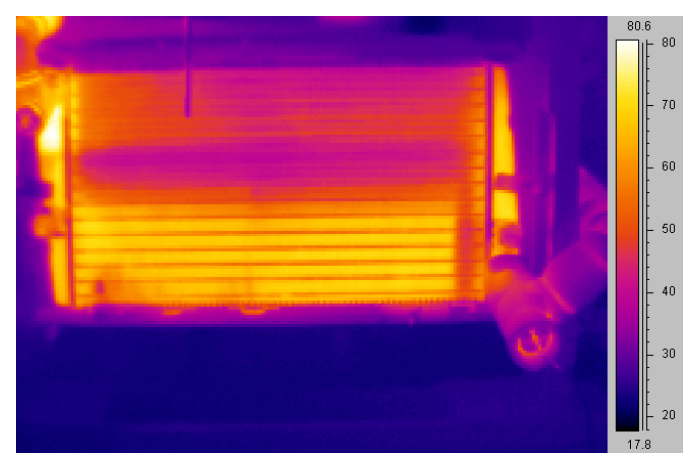

Fig. 12. The temperature field at the heat exchanger outlet cross-section - case P3-1, infrared measurement

A blocking of the air inflow to the heat exchanger causes the drop of the total heat flux of course and affects the exchanger thermal efficiency. But in some types of car coolers this method is used for regulation of the water outlet temperature, especially in winter conditions - a special blinds are applied and the exchanger outlet temperature field may look as presented in figure 12.

\section{Final remarks and conclusions}

The results of the validation procedure presented here are an effect of the initial analysis which is aimed to evaluate the HEWES code. These results show a significant discrepancies between experimental and numerical outcomes. The validation of the HEWES code is in progress. The measuring errors influence should be investigated and also the radiation heat transfer seems to be considered too. A special experiments are planned to be introduced into the validation procedure.

Application of the infrared thermography technique allowed to widespread the range of the HEWES code validation and it will be continuously used in further investigations. These measurements shown also very small flow of water in at least two pipes. This problem is probably caused by blocking of the pipes and should be fixed before continuation of the experiments. Furthermore, the pipes choking may affect the water velocity field and may be a reason of the differences between experimental and numerical results as well.

The temperature field in the heat exchanger elements identified by means of the infrared thermography may be also used for evaluation of thermal stress.

The general aim of this work was to evaluate the influence of a disturbance in the air inflow on the heat exchanger operation efficiency, so it should be stated here that blocking of the air flow by the exchanger can seriously decrease the total heat flux transported between the agents. Similar results were obtained by means of numerical simulations.

\section{Acknowledgement}

This work has been realized in the frameworks of the project No. 3T10B06229 financed by the Ministry of Science and Information Technology (at the moment Ministry of Science and Higher Education). 


\section{REFERENCES}

[1] Kostowski E. Heat Transfer. The Silesian University of Technology Publishing Office, Gliwice 2000. (in Polish)

[2] Saboya F.E.M., Sparrow E.M. Local and Average Transfer Coefficients for One-Row Plate Fin and Tube Heat Exchanger Configurations. ASME Journal of Heat Transfer, 96 (1974) 265-272.

[3] Piatek R. Thermal Analysis of Plate Fin and Tube Heat Exchanger with Unequal Inlet of Mediums. PhD Thesis, Institute of Thermal Technology, Sielsian University of Technology, Gliwice 2003. (in Polish)

[4] Bury T., Składzień J. The Experimental and the Numerical Analysis of a Ribbed Heat Exchanger With an Unequal Inlet of the Air. In Proceedings of Heat Transfer and Renewable Sources of Energy 2006, Międzyzdroje, Poland, September 13-16, 2006, 419-426.

[5] Bury T., Piątek R., Składzień J. Experimental validation of the numerical thermal model of the cross-flow heat exchanger with unequal agent flow. ECOS 2008, to be published. 
http://dx.doi.org/10.21611/qirt.2008.07_05_11 